\title{
PERSPECTIVAS ACERCA DE LAS COMPETENCIAS PROFESIONALES: RETOS PARA LA EDUCACIÓN EN TRABAJOSOCIAL
}

\author{
Ursula Manfredo Plicet ${ }^{1}$
}

\section{Resumen}

La profesión de trabajo social se enfrenta a una redefinición en cuanto a cómo afronta el desarrollo de competencias desde la educación en trabajo social y en la educación continua de sus profesionales, en armonía con el contexto del siglo XXI. Se presentan ejemplos en la educación superior en general sobre la producción de trabajos acerca del currículo fundamentado en competencias, que han dado pie a esta re-definición. La profesión de trabajo social, al igual que el concierto de las profesiones, necesita incorporarse en estas corrientes curriculares a tono con los desafíos de este tiempo. En este escrito transito a través de varias fuentes del saber que abonan a este planteamiento, las perspectivas de los participantes de la acción curricular. Estos son: la UNESCO${ }^{2}$, la $\mathrm{FITS}^{3}$, el $\mathrm{CSWE}^{4}$, el Colegio de Trabajadores Sociales de Puerto Rico, representado por el Código de Ética. Algunas aproximaciones al currículo basado en competencias, mis experiencias como investigadora cualitativa y como practicante en el servicio público; así como en la docencia que incluye la fase investigativa en la sala de clases. Finalmente, se incluyen algunas reflexiones en torno a la acción inmediata y futura, que trasciende los muros de la universidad.[Descriptores: competencias, educación en trabajo social, educación superior, currículo basado en competencias].

${ }^{1}$ Directora de la Oficina de Adiestramiento y Garantía de Calidad de la Administración de Familias y Niños del Departamento de la Familia. tural Organization.

${ }^{2}$ Siglas en ingles para United Nations Educational, Scientific and Cul-

${ }^{3}$ Siglas en español de la Federación Internacional de Trabajo Social.

${ }^{4}$ Siglas en inglés para el Council on Social Work Education. 


\section{Abstract}

The profession of social work faces a re-definition in harmony with the context of the 21st century. Higher education in general has been an abundant production of works about this re-definition. The profession of social work as well as the concert of the professions needs to incorporate in these currents in tune with the challenges of this time. This article transit through various sources of knowledge that add the perspective of curricular action participants. These are: UNESCO, the FISW, the CSWE, and the College of Social Workers of Puerto Rico, represented by the Code of Ethics. Approaches to the curriculum based on skills, use as a reference the experiences of Latin American universities and the Caribbean with curriculum based in competencies; my experience as a qualitative researcher and as a practitioner at the public services; as well as teaching that includes the research phase in classroom. Finally, some reflections are included focused in present and future actions, which go beyond the university wall. [Keywords: Competencies, Social work education, higher education, curriculum based on competencies].

Un reto, un desafío, una amenaza...

El trabajo social profesional se enfrenta en este momento histórico a varios desafíos... ...El análisis crítico de las funciones que desempeñamos como profesionales del comportamiento humano es vital para el desarrollo de nuestra pertinencia a la realidad nacional en que se enmarca nuestro trabajo... ...El desarrollo de la conciencia crítica no ha caracterizado nuestra educación formal, incluyendo la educación en trabajo social.

Dra. Raquel Seda Rodríguez ${ }^{5}$

Esta elocuente reflexión sirve de pauta para establecer la necesidad que existe en la profesión de trabajo social, de abrir la discusión a las tendencias contemporáneas en la educación superior y en los programas sociales, así como los aspectos de la educación y servicios, que se nutren recíprocamente. Es un punto de partida para abordar la tesis del desarrollo de las competencias, que me propongo discutir en este artículo.

\section{La educación en trabajo social como proceso educativo}

La educación en trabajo social, al igual que todo proceso educativo, se vincula con el desarrollo humano como ser integral y de la sociedad en general. Es por eso que, la tendencia desde las pasadas décadas, ha sido plantearse que la educación

${ }^{5}$ Fragmentos de la Ponencia: Retos al trabajo social puertorriqueño (2009). La doctora Raquel Seda Rodríguez, profesora jubilada de la Escuela Graduada de Trabajo Social, Beatriz Lassalle, Facultad de Ciencias Sociales, Universidad de Puerto Rico, Recinto de Rio Piedras. 
debe ser vista como el tesoro que encierra en sí misma. Al respecto, la Comisión Internacional sobre la Educación para el siglo XXI (1996) plantea la pregunta: "la educación o la utopía necesaria" frente al reconocimiento de los numerosos desafíos de la sociedad por venir y el reconocimiento de que la educación es un instrumento indispensable para que la humanidad pueda progresar hacia los ideales de paz, libertad y justicia social. Desde esos mismos ideales la base ético - valorativa del trabajo social como profesión, encuentra que esos mismos principios rigen la conducta ética de los profesionales de trabajo social (Código de Ética de los profesionales de Trabajo Social, 2011). Además, convergen porque la educación es la vía al servicio de un desarrollo humano más armonioso, para hacer retroceder la pobreza, la exclusión, las incomprensiones, las opresiones y las guerras, entre otros detractores de la humanidad.

La Comisión Internacional sobre la Educación para el siglo XXI (1996), en su informe recalcaron que la educación es un clamor por el amor por la infancia, por la juventud que tenemos que integrar en nuestras sociedades en el lugar que les corresponde, el sistema educativo, en la familia, en la comunidad de base, en la nación Se partió de un marco futurista tomando en cuenta la preminencia de la mundialización y procuraron delinear orientaciones válidas a nivel nacional y a escala mundial. Esta preminencia de la mundialización ha traído como resultado el aumento en el desempleo, las desigualdades y del fenómeno de la exclusión social. Ante este gran desafío es nuestra responsabilidad ética reflexionar y actuar de manera vigorosa con la educación en trabajo social. Esta educación debe ser una comprometida con el respeto de la condición humana, enfocados en el estudio serio de las condiciones sociales y económicas presentes en nuestro hemisferio geográfico y por ende en Puerto Rico, con su historia singular como nación, pueblo en el Caribe, su relación con los Estados Unidos de Norte América y su descendencia hispana.

Retomemos las palabras de Raquel Seda Rodríguez (2009), con relación al análisis crítico de las funciones que desempeñamos como profesionales del comportamiento humano que es vital para el desarrollo de nuestra pertinencia a la realidad nacional en que se enmarca nuestro trabajo. Mi propuesta es, de un trabajo de acuerdo a la sociedad cambiante. Un trabajo que se guíe por la palabra funciones que es sinónimo de actuar, desempeñar, para el que tenemos que formar trabajadores sociales bajo la mirada de la ruptura epistemológica de la educación tradicional, de la dominación ideológica de que somos objeto los mismos docentes y de la reproducción social (Morrow $\&$ Torres, 2002). En ese mismo sentido hablo de una educación 
en trabajo social contextual del siglo XXI con sus matices de mundialización con la apertura a nuevas fuentes de conocimiento endógeno y en nuestro caso, puertorriqueño. Clamo por una educación activa, con significado de las situaciones cotidianas de la vida en sociedad y que prepare al estudiantado de trabajo social a enfrentar esos desafíos que hemos estado mencionando.

La ruptura epistemológica en el estudiantado de trabajo social ocurrirá, cuando el propio docente debe hacer un ejercicio de mirarse hacia adentro para situar su visión, sus límites y sus prácticas educativas. Hay que ser un educador integral para formar futuros trabajadores y trabajadoras sociales completos. Ser un aprendiz del alcance del constructo competencia para estar en posición de enseñarlas. El desempeño de las funciones va de la mano con las competencias o conjuntos de habilidades, conocimientos, actitudes y valores y es necesario que un alumno vaya adquiriéndolas y desarrollándolas a lo largo de su formación universitaria, ya que se consideran imprescindibles para cualquier profesional que pretenda trabajar en una sociedad como la actual (Sanz de Acedo Lizárraga, 2010). Por su parte, abona a este planteamiento Aventín en Blanco (2009), cuando reconoce que la competencia está ligada al desempeño profesional. La competencia no existe en sí misma, es decir, separada de una actividad, de la solución de un problema de un contexto determinado, que también pueda ser transferible a otras experiencias.

Es probable que algunos colegas de trabajo social no estén de acuerdo con esta manera de proponer los conceptos anteriores. Esto es comprensible, porque existen múltiples realidades o distintos puntos de vista sobre la realidad que se estudia. El planteamiento central de este artículo se refiere a los lineamientos internacionales para la educación superior y la educación en trabajo social de la cual no podemos sustraernos en nuestra sociedad influenciada de esa realidad mundializada, tan cambiante.

Abordajes para la educación universitaria desde otras
visiones, síntesis y pistas de organizaciones internacionales
Derrida (1984), propone la "problematización" de la
universidad cuando escribe que la universidad no se compone
de sólo muros y estructuras exteriores que la rodean y protegen
nuestro trabajo, sino también es la estructura de nuestra
interpretación. Mi interpretación es que la educación es un
instrumento para el cambio social. Ésta no debe verse como
un instrumento de garantía para la reproducción cultural o
para institucionalizar el cambio o la innovación para perpetuar,
precisamente, las estructuras del Estado o las visiones


dominantes de los académicos, según su preferencia ideológica. De otra parte, Escotet (2004) plantea que:

\begin{abstract}
No hay duda que la institución de educación superior por sí misma no puede hacer frente a un desafío que corresponde a toda la sociedad. Pero si alguna institución debería ser rectora en contribuir a balancear el pensamiento utópico y pragmático, renovar la práctica democrática, a formar el sentido ético y estético de la sociedad, a ser motor de una nueva renovación del espíritu, esa institución es la universidad (p. 17).
\end{abstract}

Sin lugar a dudas la educación en trabajo social necesita de una reforma que la posicione de tal forma que pueda transitar hacia modelos de práctica sensibles a la realidad social del siglo XXI. En primer lugar, los docentes de las distintas unidades académicas deben ser protagonistas de esta reforma, asumiendo responsabilidad por su desarrollo profesional acerca de cómo aprende a enseñar y se enseña a aprender (Manfredo Plicet, 2007). En segundo lugar, mediante el desarrollo de iniciativas de investigación rigurosas que informen la práctica profesional desde el contexto local. Tercero, utilizar la universidad como plataforma para estimular y encausar en el estudiantado el deseo por aprender, la curiosidad intelectual y el aprendizaje a lo largo de la vida en todos los ámbitos culturales del saber. Finalmente, el posicionamiento de la universidad en cuanto al desarrollo de nuevo conocimiento, su misión social como garante de los valores universales y del patrimonio cultural. Más allá de la idea tradicional que la universidad por lógica formaba profesionales (Ortega y Gasset, 1936), para administrar la cosa pública y el mercado laboral. En fin, "desde la misión de la universidad del siglo XXI, la profesión de trabajo social al igual que otras, en el concierto de las profesiones, incluye creación de conocimiento, desarrollo de responsabilidad social y el servicio a la sociedad" (Manfredo Plicet, 2007, p.10).

Respalda esta discusión lo planteado por Malaclaza (2003), que la sociedad se transforma radicalmente, producto del interjuego de las relaciones sociales, por la relación del Estado y la sociedad civil. Señala esta autora que esto coloca en una posición de vulnerabilidad a las unidades académicas de trabajo social, que es necesario analizar profundamente para generar cambios necesarios y revertir resultados adversos por la falta de inacción.

\title{
Síntesis de las pistas de la UNESCO
}

Inserto en la discusión una síntesis de las pistas y recomendaciones y de los cuatro pilares de la educación 
propuestos por la Comisión Internacional sobre la Educación para el siglo XXI a la UNESCO (1996). La educación tiene un papel muy concreto en llevar a cabo la tarea universal de comprender mejor el mundo, comprender al otro, para comprenderse mejor a sí mismo. Por su parte, la política educativa debe ser amplia, con una visión diversificada que no quede espacio para la exclusión intelectual de aquellas personas que no compartan nuestras visiones. La educación a lo largo de la vida se basa en cuatro pilares: aprender a conocer, aprender a aprender, aprender a vivir juntos, aprender a ser.

Aprender a conocer, combinando una cultura general suficientemente amplia con la posibilidad de profundizar los conocimientos en un pequeño número de materias, lo que supone además, aprender a aprender para aprovechar las posibilidades que ofrece la educación a lo largo de la vida.

- Aprender a hacer, a fin de adquirir no solo una calificación profesional sino, más generalmente, una competencia que capacite al individuo para hacer frente a gran número de situaciones y a trabajar en equipo. Pero también, aprender a hacer en el marco de las distintas experiencias sociales o de trabajo que se ofrece a los jóvenes y adolescentes, bien espontáneamente a causa del contexto social o nacional, bien fundamentalmente gracias al desarrollo de la enseñanza por alternancia.

- Aprender a vivir juntos, desarrollando la comprensión del otro y la percepción de las formas de interdependenciarealizar proyectos comunes y prepararse para tratar los conflictos - respetando los valores de pluralismo, comprensión mutua y paz.

- Aprender a ser, para que florezca mejor la propia personalidad y se esté en condiciones de obrar con creciente capacidad de autonomía, de juicio, y de responsabilidad personal. Con tal fin, no menospreciar en la educación ninguna de las posibilidades de cada individuo: memoria, razonamiento, sentido estético, capacidades físicas, aptitud para comunicar (Comisión Internacional sobre la Educación para el siglo XXI a la UNESCO, 1996).

Autoras como Sanz de Acedo Lizárraga (2010), presentan el alcance del constructo competencia y parte de los cuatro pilares propuestos por la Comisión Internacional sobre Educación (1996). Resalta la convergencia en la identificación e importancia de la inclusión de las competencias de Sanz de Acedo Lizárraga con lo propuesto por esta autora.

Con la finalidad de continuar ilustrando las tendencia del currículo contemporáneo utilizaré de referente los trabajos 
realizados por un grupo de educadores de América Latina y del Caribe, representado por docentes de universidades como: Universidad Nacional de Cuyo (Argentina), Universidad de Talca (Chile), Universidad de Caldas (Colombia), Universidad del Norte(Colombia), Universidad Industrial de Santander (Colombia), Universidad del Valle (Colombia), Pontificia Universidad Javeriana (Colombia), Universidad de Costa Rica (Costa Rica), Escuela Superior Politécnica del Litoral (Ecuador), Universidad de Monterrey (México), Pontificia Universidad Católica del Perú (Perú), Universidad Peruana Cayetano Heredia (Perú), Universidad del Pacífico (Perú) y el Instituto Tecnológico de Santo Domingo INTEC (República Dominicana). Titulado: Currículo basado en Competencias, en las Memorias del Seminario Internacional Universidad del Norte, Barranquilla, Colombia (25 y 26 de julio de 2005) del mismo he tomado unas ideas para ilustrar el tono de la discusión acerca del currículo basado en competencias. En primer lugar, veamos cómo este grupo de educadores definieron el concepto de competencia (Currículo universitario ...(2005):

\begin{abstract}
Por competencias se entiende la concatenación de saberes, no sólo pragmáticos y orientados a la producción, sino aquellos que articulan una concepción del ser, del saber, saber hacer, del saber convivir. Esto significa que frente a una situación dada quien tiene la competencia para actuar posee los conocimientos requeridos y la capacidad para adecuarlos a las condiciones específicas, tiene las habilidades para intervenir eficaz y oportunamente y está imbuido también de los valores que le permite asumir actitudes acordes con sus principios y valores. (p.9)
\end{abstract}

Sobresale de esa cita, que sus autores insertan los mismos ideales de los cuatro pilares de la educación, propuestos por la Comisión Internacional sobre la Educación para el siglo XXI a la UNESCO (1996).

En el campo de la profesión de trabajo social, una organización de prestigio internacional es la Federación Internacional de Trabajo Social. Tiene el carácter de ser rectora en agrupar organizaciones profesionales nacionales de trabajo social y además en organizaciones como las Naciones Unidas y la Organización Internacional del Trabajo (OIT).

\title{
La Federación Internacional de Trabajadores Sociales
}

La historia de la Federación Internacional de Trabajadores Sociales (FITS) se puede trazar como la sucesora del Secretariado Internacional Permanente de Trabajadores Sociales. Fue fundada en París en 1928. La FITS de hoy, se ubica en Múnich en 1956, 
como una organización de trabajadores sociales profesionales. La FITS (2002), definió la profesión de trabajo social de la manera siguiente:

Current definition: The social work profession promotes social change, problem solving in human relationships and the empowerment and liberation of people to enhance well-being. Utilizing theories of human behavior and social systems, social work intervenes at the points where people interact with their environments. Principles of human rights and social justice are fundamental to social work (cf.).

La representación de la FITS en organizaciones internacionales se manifiesta de la siguiente manera: uno, se distingue por el rol de consultor especial en el Consejo Económico y Social de las Naciones Unidas y el Fondo de las Naciones Unidas para la Infancia. Existen grupos representativos de la FITS que trabajan, actualmente, en las Naciones Unidas en Ginebra, Nueva York y Viena; y dos, la FITS está también en la lista especial de Organizaciones No-Gubernamentales de la OIT.

En la revisión de esta fuente de referencia, encontré que uno de los proyectos que despunta en la FITS, es el mejoramiento de la enseñanza del trabajo social. Precisamente, uno de los propósitos de este trabajo, es contribuir en la discusión sobre la educación en trabajo social contextual al siglo XXI y, por ende, en Puerto Rico. Esto, resulta pertinente para traer en esta discusión algunas ideas sobre las políticas educativas de la educación universitaria y las del Consejo Educativo de Trabajo Social, conocidas por sus siglas en inglés como el CSWE.

\section{Las políticas educativas del Consejo Educativo de Trabajo Social narrativa de los antecedentes profesionales: educación en servicio}

Desde mis años de estudios doctorales en educación, como trabajadora social en la docencia y en funciones en programas sociales a nivel público, en escenarios de salud mental, abuso de sustancias, servicios sociales, entre otros; sentí un gran interés en estudiar los procesos educativos de la profesión en trabajo social. Se despierta en mí este interés, por el contacto directo en el adiestramiento institucional - sistémico de más de 2,400 trabajadores sociales y luego además, paso a asumir la responsabilidad de crear y dirigir la Oficina de Garantía de Calidad para el programa de bienestar del menor.

En ambas instancias pude enfrentar con documentación empírica, la apremiante necesidad de desarrollar de manera continua, el personal de trabajo social en las distintas capacidades de servicio directo, de supervisión, de gerencia o en los aspectos 
normativos, a nivel central, en la que estos pudieran articular la teoría con la práctica, fundamentada en el modelo de práctica adoptado en la Agencia. Esta realidad me llevó a re-plantearme los contenidos, las prácticas educativas, la forma como valoramos los aprendizajes, entre otros, en la formación de trabajadores sociales que pudieran transferir los conocimientos, las destrezas y las actitudes. Es decir, un conjunto de aptitudes o de competencias a los lugares de trabajo social con una multiplicidad de sistemas humanos. Esta situación produjo en mí la curiosidad intelectual de hacer uso de una combinación de la teoría y la práctica en trabajo social de los fundamentos, la innovación y la creación de conocimiento en educación de cara al siglo XXI.

Una muestra de esto, fueron los variados trabajos realizados, en torno al currículo, el análisis del discurso del currículo en armonía a los paradigmas crítico y constructivista, los métodos activos de enseñanza y aprendizaje, el método y los modos del liderazgo didáctico o el desarrollo continuo de los educadores para que estos puedan diseñar e implantar las prácticas educativas en la sala de clases.

Algunos ejemplos de estos contenidos y trabajos fueron: La educación de adultos, la educación superior, la investigación acción, la etnografía, en particular el proyecto sobre la comunidad de aprendices (Manfredo Plicet, 2004), el desarrollo de videos sobre el Centro de Infantes y Maternales (se refiere al desarrollo humano), el internado en el Departamento de Trabajo Social (sub - graduado) de la Facultad de Ciencias Sociales de la Universidad de Puerto Rico en Río Piedras, hasta la disertación doctoral (Manfredo Plicet, 2007). En el Departamento de Trabajo Social tuve la oportunidad de relacionarme con el proceso de auto - estudio para la re-acreditación ante el CSWE y desarrollar instrumentos para el avalúo del aprendizaje.

En este empeño, por adentrarme en la comunidad de aprendices desde mi propia definición como investigadora, tuve la oportunidad de relacionarme con dos auto-estudios realizados por la facultad de la Escuela de Trabajo Social, para la re-acreditación ante el CSWE, en dos periodos. Esto, me permitió estudiar a fondo el paradigma del currículo, el currículo, los contenidos, los recursos humanos, entre otros. Culminados mis estudios doctorales en educación, he tenido la oportunidad de ofrecer cursos en trabajo social a nivel sub - graduado y graduado en varios centros universitarios del país.

Asimismo, he continuado mis funciones en el servicio público lo que me permite como "insider" valorar lo enseñado en la sala de clases y lo que se necesita en los programas sociales, en el contexto del siglo XXI. Todo ello, sin perder de perspectiva 
que una buena parte de los fondos federales son delegados a organizaciones privadas de base comunitaria. Este conocimiento de primera mano me lleva a reflexionar, en este contexto, sobre la utilidad de hacer uso de competencias educativas en los procesos formadores del estudiantado de trabajo social.

En relación a esta tesis que estoy planteando acerca de las competencias profesionales, en la que he insertado el constructo de competencias y las competencias educativas del CSWE, he escuchado algunas voces disonantes que entienden que las competencias representan la dominación ideológica de un contexto capitalista neo-liberal. Sobre esta discusión, introduzco a Derrida (2000) quién postula la idea de que la universidad es una filosófica, a saber, la idea de la pirámide del saber, de regiones y de disciplinas, dominadas por la idea de un saber absoluto, un saber del saber que es la universidad. En la entrevista: El porvenir de la profesión o la Universidad sin condición, planteó algunas ideas acerca del imperialismo americano, y tomó como ejemplo su propia experiencia. El sólo sabia escribir en francés, pero le interesó que tradujeran sus escritos en el idioma inglés, para que en el Japón, la India y en muchos otros países sus escritos produjeran,

\begin{abstract}
...efectos en inglés de un pensamiento transformado o lo que se esconde detrás de un texto francés que resiste al inglés. Es de esta manera que uno hace una obra, si uno hace una obra tomando la lengua del dominador, si se puede decir, la lengua imperial o imperialista. Uno la toma y trata de subvertirla como uno pueda haciendo que hable de otra manera. Haciendo que en el interior mismo de la lengua americana proteste una cierta hegemonía americana (Derrida, 2000, p.4).
\end{abstract}

Desde este argumento invito a profesionales y facultad de trabajo social a que nos expongamos al constructo de las competencias del dominador para subvertir la dominación desde la lengua del dominador.

\title{
Políticas educativas del CSWE del 2008: Competencias ${ }^{6}$
}

Un conjunto de diez competencias y conductas prácticas fueron adoptadas por el CSWE, a partir del 2008. Con la finalidad de ilustrar con ejemplos en esta discusión, una selección de las mismas y de conductas observables se presenta a continuación.

${ }^{6}$ Traducción libre realizada por la Dra. Elizabeth Miranda, Directora de la Escuela de Trabajo Social, Universidad Inter Americana - Recinto Metro. 
Figura 1. Resumen de las políticas educativas, la descripción de la competencia y la conducta observable.

\begin{tabular}{|c|c|c|}
\hline $\begin{array}{l}\text { Política } \\
\text { educativa }\end{array}$ & Descripción de la competencia & Conducta observable \\
\hline 2.1 .1 & $\begin{array}{l}\text { Se identifica con la profesión de } \\
\text { trabajo social y mantiene una conducta } \\
\text { profesional. }\end{array}$ & $\begin{array}{l}\text { Intercede por el acceso de los clientes a los servicios de trabajo social. } \\
\text { Está comprometido con su aprendizaje a largo plazo. }\end{array}$ \\
\hline 2.1 .2 & $\begin{array}{l}\text { Aplica los principios éticos del trabajo } \\
\text { social para guiar su práctica. }\end{array}$ & $\begin{array}{l}\text { - Reconoce y maneja sus valores personales de tal forma que sus valores profesionales guian su } \\
\text { práctica. } \\
\text { Hace decisiones éticas mediante la aplicación del Código de Ética de la NASW y según sea aplicable } \\
\text { de la Federación Internacional de Trabajadores Sociales/Asociación Internacional de Escuelas de } \\
\text { Trabajo Social en el establecimiento de principios éticos del trabajo social. }\end{array}$ \\
\hline 2.1.3 & $\begin{array}{l}\text { Aplica pensamiento crítico para } \\
\text { documentar y comunicar sus juicios } \\
\text { profesionales. }\end{array}$ & $\begin{array}{l}\text { Distingue, justiprecia e integra múltiples fuentes de conocimiento. } \\
\text { Analiza modelos de ponderación, prevención, intervención y evaluación; y demuestra tener una } \\
\text { comunicación oral y escrita efectiva en el trabajo con individuos, familias, grupos, organizaciones, } \\
\text { comunidades y colegas. }\end{array}$ \\
\hline 2.1 .5 & $\begin{array}{l}\text { Aboga por los derechos humanos y la } \\
\text { justicia social y económica. }\end{array}$ & $\begin{array}{l}\text { Comprende las formas y mecanismos de opresión y discriminación. } \\
\quad \text { Intercede por los derechos humanos y la justicia social y económica. } \\
\text { Se involucra en prácticas para promover la justicia social y económica. }\end{array}$ \\
\hline 2.1 .6 & $\begin{array}{l}\text { Se involucra en una práctica } \\
\text { documentada por la investigación, en } \\
\text { una investigación documentada por la } \\
\text { práctica. }\end{array}$ & $\begin{array}{l}\text { - Usa la experiencia práctica para informar la investigación científica. } \\
\text { - Usa la investigación para informar su práctica. }\end{array}$ \\
\hline 2.1 .7 & $\begin{array}{l}\text { Aplica conocimientos de conducta } \\
\text { humana y el ambiente social. }\end{array}$ & $\begin{array}{l}\text { - Utiliza los marcos teóricos conceptuales para guiar los procesos de ponderación, intervención y } \\
\text { - } \quad \text { Craluación. } \\
\text { - } \quad \text { y aplica conocimientos para entender la persona y su ambiente. }\end{array}$ \\
\hline 2.1 .8 & $\begin{array}{l}\text { Se involucra en la práctica de la politica } \\
\text { social. }\end{array}$ & $\begin{array}{l}\text { Analiza, formula e intercede en la promoción del bienestar social. } \\
\text { - Colabora con colegas y clientes en acciones políticas efectivas. }\end{array}$ \\
\hline 2.1 .10 (a) & $\begin{array}{l}\text { Establece una relación profesional, } \\
\text { pondera, interviene y evalúa individuos, } \\
\text { familias, grupos, organizaciones y } \\
\text { comunidades. }\end{array}$ & $\begin{array}{l}\text { - La práctica profesional incluye procesos interactivos y dinámicos con múltiples sistemas humanos. } \\
\text { Usa la investigación y los adelantos tecnológicos, los resultados de la evaluación de programas, la } \\
\text { efectividad de la práctica, el desarrollo de análisis y la intercesión. Asume liderazgo en politicas y } \\
\text { servicios; y en la promoción de la justicia social. }\end{array}$ \\
\hline
\end{tabular}


Un vistazo al resumen de las competencias en la Figura 1, reflejan la congruencia con el contenido de los documentos del FITS. Esto queda reconfirmado en el texto siguiente: "The International Federation of Social Workers (IFSW) is a global organization striving for social justice, human rights and social development through the promotion of social work, best practice models and the facilitation of international cooperation" (cf. http://ifsw.org/what-we-do/).

Además, estos documentos desde el punto de vista educativo y de la razón de ser de la Federación Internacional de Trabajo Social que agrupa a más de 86 organizaciones miembros a nivel internacional; se encuentran muy cercanos para ofrecer respuesta al planteamiento sobre de la necesidad de desarrollar la conciencia crítica. Aspecto en la educación en trabajo social, al que Seda Rodríguez (2009) apuntaba en la siguiente cita: "El desarrollo de la conciencia crítica no ha caracterizado nuestra educación formal, incluyendo la educación en trabajo social", texto que cité en párrafos anteriores.

\section{Aproximaciones al currículo en trabajo social basado en competencias}

Es vinculante en esta discusión plantearnos la necesidad de desarrollar la educación en trabajo social en un currículo basado en competencias. En términos generales, en las carreras universitarias las competencias del estudiante sub - graduado y graduado, no sólo estriban de las instancias laborales, sino también en otros aspectos, de los que la educación en trabajo social no es la excepción. Veamos: (1) el proyecto educativo institucional universitario, (2) de las tendencias nacionales, esto incluye mirar críticamente el contexto en el que se practica la profesión de trabajo social y por ende en Puerto Rico, (3) las tendencias internacionales de la profesión, (4) de los avances científicos en todos los campos del saber, de los cuales también se nutre la profesión de trabajo social y (5) de la movilidad geográfica mundializada a la que están sujetos los estudiantes y su entorno familiar.

Cuando definimos las competencias del graduado en el nivel de enseñanza a nivel superior, me refiero al nivel sub - graduado y graduado en trabajo social (con las diferencias reconocidas en cuanto a profundidad del generalista o avanzado), se ha de considerar el contexto internacional, tales como las agencias acreditadoras, las asociaciones profesionales, organismos internacionales, entre otros. El contexto nacional lo forman los colegios profesionales, otros centros de educación superior, el contexto institucional se refiere a los planes de desarrollo institucional, a los planes específicos de cada facultad. En el área de las distintas disciplinas profesionales, que implica tomar en consideración aspectos como los resultados 
de las investigaciones, revistas especializadas, opinión de científicos e investigadores.

Hay que establecer las diferencias fundamentales entre un currículo basado en competencias y el currículo tradicional perenne, del que se discute ampliamente en la literatura en educación. El primero se refiere a la planificación que se hacen los participantes de la acción curricular, a partir de las necesidades prospectivas de la realidad contextual en la que se va a desempeñar el estudiante. Por el contrario, el currículo tradicional perenne, se planifica desde la lógica conceptual que establecen los académicos, sin implicaciones de revisiones cíclicas, distanciado de la realidad contextual en el que opera la institución educativa. Con esto en mente, presento parte de mi experiencia en la sala de clases.

Un ejemplo concreto en la sala de clases: mi narrativa acerca del currículo en trabajo social basado en competencias

"Tengo que admitir que me sacó de la zona de confort" (Extracto del Diario Reflexivo de estudiante del Curso Comportamiento Humano II, 2010), este es un testimonio de una estudiante con respecto a su experiencia de aprendizaje mediante prácticas activas que desarrollé.

Antes de iniciar la discusión de esta sección del escrito, mostraré el diagrama del reflexivo que desarrollé con el propósito de sintetizar los aspectos de política institucional, organismos acreditadores, prontuario del curso, práctica educativa, ejercicios, entre otros.

de clases $^{7}$

Figura 2. Aspectos que nutren el currículo en la sala

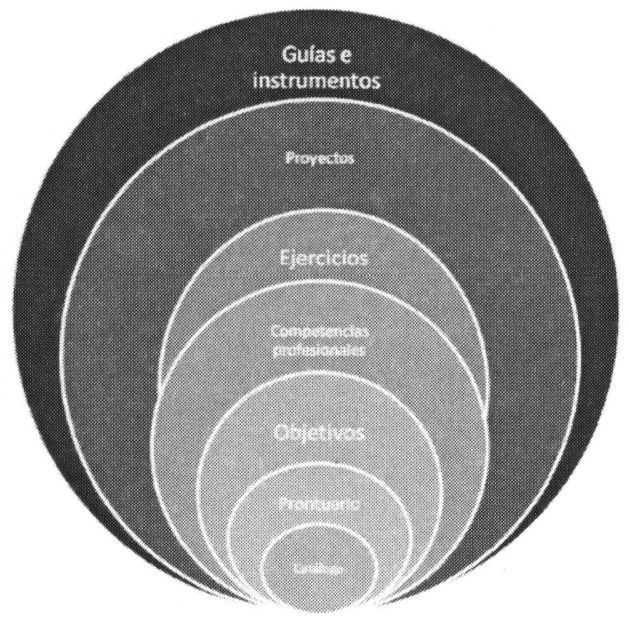

${ }^{7}$ Conceptuado por Dra. Úrsula Manfredo Plicet ( $)$ (2010) 
Las competencias profesionales a desarrollar en el estudiantado identificadas en este curso fueron las siguientes:

2.1.2 Aplica los principios éticos del trabajo social para guiar su práctica.

2.1.7 Aplica conocimientos sobre la conducta humana y el ambiente social.

Como se puede apreciar, este es un proceso integrador, vinculante, pensado para el desarrollo de las competencias del estudiantado en trabajo social. Los procesos de enseñanza aprendizaje estuvieron matizados por una reflexión continua de la profesora y del estudiantado, para este propósito desarrollé instrumentos de avalúo del aprendizaje como los diarios reflexivos y rúbricas.

La rúbrica que desarrollé en el 2010, fue adaptada para fines de este artículo. El documento original incluye las dos competencias mencionadas en las figuras anteriores. Queda demostrado en esta discusión el reto que plantea la educación en trabajo social en este tiempo. En la formación de profesionales en trabajo social, es ineludible realizar cambios paradigmáticos, epistemológicos, metodológicos, didácticos y de competencias docentes o lo que he llamado el desarrollo continuo del educador o liderazgo didáctico (ManfredoPlicet, 2007). También, que estos cambios en las prácticas de enseñanza deben promover la participación, la cooperación y estimular el pensar del estudiantado, en la medida que se construyen los conocimientos junto al docente, repensar sobre lo aprendido o la meta cognición del estudiantado, tal como lo esbocé en la comunidad de aprendices (ManfredoPlicet, 2004/07). Esto es posible de alcanzar con un currículo basado en competencias con énfasis en el desarrollo del pensamiento crítico. 
Figura 3. Rúbrica para valorar los aprendizajes en el curso ${ }^{8}$

\begin{tabular}{|c|c|c|c|c|c|c|}
\hline \multirow{4}{*}{ 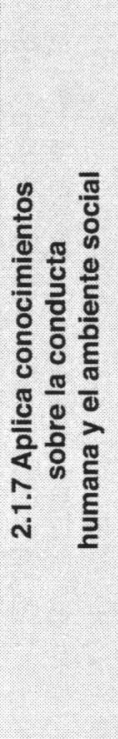 } & \multirow{2}{*}{ Conducta observable } & \multirow{2}{*}{$\begin{array}{c}\text { Resumen } \\
\text { de la } \\
\text { puntuación }\end{array}$} & \multicolumn{4}{|c|}{ Descriptores de la conducta observable } \\
\hline & & & $\begin{array}{l}\text { Consisten-temente } \\
\text { (4) }\end{array}$ & $\begin{array}{c}\text { General-mente } \\
\text { (3) }\end{array}$ & $\begin{array}{c}\text { Alguna veces } \\
\text { (2) }\end{array}$ & $\begin{array}{l}\text { Demuestra } \\
\text { inicial } \\
\text { (1) }\end{array}$ \\
\hline & $\begin{array}{l}\text { Utiliza los } \\
\text { marcos teóricos } \\
\text { conceptuales para } \\
\text { guiar los procesos } \\
\text { de ponderación, } \\
\text { intervención y } \\
\text { evaluación. }\end{array}$ & & & & & \\
\hline & $\begin{array}{l}\text { Critica y aplica } \\
\text { conocimientos para } \\
\text { entender la persona y } \\
\text { su ambiente. }\end{array}$ & & & & & \\
\hline
\end{tabular}

${ }^{8}$ Comportamiento Humano II @ Manfredo Plicet (2010/12) 


\section{Retos en torno a la acción inmediata y futura}

La educación en trabajo social actual, amerita una reflexión profunda, mediante el análisis riguroso de las distintas fuentes del saber. Con una práctica inclusiva de todos los sectores, o lo que se define en el currículo como los participantes de la acción curricular. Es por eso, que en este escrito he transitado en diversas fuentes como la UNESCO, la FITS, el CSWE, el Colegio de Trabajadores Social de Puerto, escrito de reconocidos trabajadores sociales, además de mi experiencia en una agencia pública de servicios sociales y en la academia, entre otros.

Por el contrario, una sola visión procedente de la lógica de los académicos, no responde a la existencia de conflictos y a la multiplicidad de los sistemas humanos. La educación en trabajo social en el presente siglo, necesita de hacedores de políticas sociales, para que su voz sea escuchada, administradores con dominio en gestiones gubernamentales, que aseguren la transparencia en los procesos, de gerencia media, así como de servicios directos, que puedan transitar de la política social a la implantación comprometida de la misma. De igual modo, hace falta profesionales de trabajo social con las competencias en la organización de la comunidad, defensores de los derechos humanos, que impulsen con acciones concretas la justicia social y económica.

En otra dimensión, emerge en la discusión la educación en trabajo social que está más allá de la culminación de un grado académico o de los muros de la universidad. Me refiero a profesionales practicantes en las distintas esferas donde nos desempeñamos. Hay un llamado urgente a mantener el apego a los valores éticos, a los principios de defensa de la justicia social y económica. Es un llamado a alzar la voz para que, a pesar de los impactos políticos partidistas, defendamos desde nuestros talleres de trabajo los fondos públicos, para que los servicios lleguen a la ciudadanía para lo que están destinados, con la calidad que la dignidad humana requiere. En otras palabras hacemos política social desde su formulación hasta cuando esta política se traduce en llevar el servicio de manera profesional, con modelos de práctica en la prevención, la educación, la intervención y la rehabilitación, así como, en la evaluación de los mismos. Hacemos política social, cuando no nos quedamos callados y nos mantenemos incólumes lanzando a la discusión los errores que comenten los que dirigen las estructuras formales.

Hay unas experiencias de aprendizajes para el desarrollo integral del estudiantado, del docente y del trabajador social en la práctica profesional, que están conjugadas en los saberes de los cuatro pilares de la educación (Comisión Internacional sobre la Educación para el siglo XXI, 1996), en cuanto a lo conceptual o saber conocer, lo procedimental o saber hacer y en lo referente a las actitudes o saber ser y aprender a vivir juntos o la vida en sociedad. Estos ideales reproducen o sintetizan la visión humanista que permea en nuestra profesión de trabajo social, no solo para nosotros, sino para la sociedad en el contexto del presente siglo. 


\section{REFERENCIAS}

Aventín, Bonsón (2009). Desarrollo de competencias en educación superior. En Ascensión Blanco (Coord.) Desarrollo y evaluación de competencias en educación superior (pp. 17-20). España: Narcea.

Código de ética de profesional de trabajo social de Puerto Rico (2011). Recuperado de http://ctspr.org/pdf/codigo_etica.pdf

Comisión Internacional sobre la Educación para el siglo XXI (1996). La educación encierra un tesoro (Informe a la UNESCO). Recuperado de:http://www.bvs.sld.cu/revistas/ems/vol16_1_02/ ems04102.htm

Currículo universitario basado en competencias. Memorias del Seminario Internacional Universidad del Norte, Barranquilla, Colombia. 25 y 26 de julio de 2005. Recuperado de: http://aula.virtual. ucv.cl/aula_virtual/cinda/cdlibros/35Curr\%C3\%ADculo\%20 Universitario $\% 20 \mathrm{Basado} \% 20$ en $\% 20 \mathrm{Competencias/}$ Curr\%C3\%ADculo\%20Universitario\%20Basado\%20en\%20 Competencias.pdf

Derrida, Jacques (2000). El porvenir de la profesión o la universidad sin condición. Revista Postdata. Recuperado de http://www. jacquesderrida.com.ar/textos/mara_negron_entrevistahtm

Derrida, Jacques (1984). La filosofía como institución (Traducción de A. Azurmendi). Barcelona: Juan Granica.

Escotet, Miguel Angel (2004). Crisis de la calidad de la enseñanza superior: desafíos ante el nuevo siglo de incertidumbre. Tendencias de la consulta regional de la UNESCO en la educación superior. Recuperado de: http://www.unesco.org/ education/educprog/wche/

Federación Internacional de Trabajo Social. Recuperado de http:// aprendeenlinea.udea.edu.co//ms/moodle/file.php/393/La_ FITS_en_Sintesis.htm.

Malaclaza, Susana.L. (2003). Desde el imaginario social del siglo XXI: Repensar el Trabajo Social. Buenos Aires: Espacio Social.

Manfredo Plicet, Ursula (2012, marzo). Tengo que admitir que me sacó de la zona de confort. Ponencia presentada en el Segundo Encuentro de Investigadores Cualitativos de la Unión Puertorriqueña de Investigadores Cualitativos, Universidad del Este, Carolina, PR.

Manfredo Plicet, Ursula (2007). El programa de maestría de la Escuela Graduada de Trabajo Social de la Universidad de Puerto Rico: Una exploración microetnográfica de la comunidad de aprendices. (Disertación doctoral no publicada). Universidad de Puerto Rico en Río Piedras, PR.

Manfredo Plicet, Ursula (2004). En búsqueda de la comunidad de aprendices: Un estudio etnográfico el Programa de Maestría de la Escuela Graduada de Trabajo Social de la Universidad de Puerto Rico. Trabajo de investigación doctoral para el curso Investigación Etnográfica. Manuscrito no publicado, Universidad de Puerto Rico en Río Piedras, PR. 
Miranda, Elizabeth (2008).Competencias: politicas educativas CSWE. Traducción libre, para fines de discusión en la Facultad ETS UIA.

Morrow, Raymond Allan. \& Torres, Carlos Alberto. (2002). Las teorías de la reproducción social y cultural. España: Editorial Popular.

Ortega y Gasett, José (1936). Misión de la Universidad: $y$ otros ensayos afines.Madrid: Ediciones Revista Occidente.

Sanz de Acero Lizárraga, Maria Luisa. (2010). Competencias cognitivas en educación superior. España: Ediciones Narcea.

Seda Rodríguez, Raquel (2009). Retos al Trabajo Social puertorriqueño. Recuperado el en http://www.arecibo.inter.edu/reserva/tsocial/ retos.pdf

The future of social work. Recuperado de:http://ifsw.org/get-involved/thefuture-of-social-work/ 